\title{
Phylogenetic Analysis of the Genus Chlamydia Based on 16S rRNA Gene Sequences
}

\author{
PUDJIATMOKO, HIDETO FUKUSHI,* YOSHITSUGU OCHIAI, \\ TSUYOSHI YAMAGUCHI, AND KATSUYA HIRAI \\ Department of Veterinary Microbiology, Faculty of Agriculture, \\ Gifu University, Gifu 501-11, Japan
}

\begin{abstract}
The phylogenetic relationships among Chlamydia spp. were investigated by comparing 16S rRNA gene sequences. In this analysis we used 14 strains of Chlamydia psittaci, including seven feline isolates, two avian isolates, two human isolates, one bovine isolate, one ovine isolate, and one guinea pig isolate; five strains of Chlamydia pecorum, including three bovine isolates, one ovine isolate, and one koala isolate; and nine strains of Chlamydia trachomatis, including six human isolates, two swine isolates, and one mouse isolate. A phylogenetic analysis of the $16 \mathrm{~S}$ rRNA gene sequences of these organisms and seven previously published sequences revealed eight genetic groups which formed two clusters. The first cluster was composed of $C$. pecorum, Chlamydia pneumoniae, and $C$. psittaci and included three genetic groups (one group containing avian, human, and ovine strains, one group containing feline strains, and one group containing guinea pig strains). The second cluster was composed of $\boldsymbol{C}$. trachomatis and also included three genetic groups (one group containing human strains, one group containing swine isolates, and one group containing rodent strains). The strains in each genetic group exhibited similar genetic distances. The results of the phylogenetic analysis agreed with the results of previous genomic DNA, ompA gene allele, and biotyping studies. Therefore, the genetic groups based on genetic distances may be considered a criterion for species identification.
\end{abstract}

Chlamydiae are obligatory intracellular bacteria that have a unique developmental cycle (25). These organisms are currently placed in the order Chlamydiales, family Chlamydiaceae, and genus Chlamydia. The following four species have been recognized in the genus: Chlamydia trachomatis, Chlamydia psittaci, Chlamydia pneumoniae, and Chlamydia pecorum $(6,14$, $25)$. Differentiation of these species is based on the following criteria: inclusion morphology, the presence of glycogen in inclusions, sulfadiazine sensitivity, and DNA-DNA homology values $(6,13,14,26)$. C. trachomatis, the type species of the genus Chlamydia, and C. psittaci were the first two species assigned to the genus and were distinguished on the basis of differences in inclusion morphology, glycogen content, and susceptibility to sulfadiazine $(13,26)$. C. pneumoniae was initially identified as $C$. psittaci, because its phenotypic characteristics are similar to those of $C$. psittaci (i.e., dense inclusions, glycogen negative, resistant to sulfadiazine). C. pneumoniae was, however, proposed as a new species on the basis of ultrastructural differences in the morphology of its elementary bodies, its levels of DNA-DNA homology with other chlamydiae, and its serological reactivity (14). C. pecorum, the fourth species, was also placed in $C$. psittaci on the basis of its phenotypic characteristics and was separated from $C$. psittaci on the basis of DNA-DNA homology values and immunological specificities $(6,7)$.

C. psittaci contains a variety of chlamydiae with different genetic, serological, and host-tropic properties. Our previous DNA-DNA hybridization analysis revealed levels of homology ranging from 14 to $95 \%$ between $C$. psittaci strains (5). The levels of DNA homology between mammalian strains and avian strains of $C$. psittaci were less than $70 \%$; the mammalian strains could be separated into two groups, feline strains and

\footnotetext{
* Corresponding author. Mailing address: Department of Veterinary Microbiology, Faculty of Agriculture, Gifu University, Yanagido 1-1, Gifu 501-11, Japan. Phone: 81-58-293-2946. Fax: 81-58-293-2947. E-mail: hfukushi@cc.gifu-u.ac.jp.
}

ovine abortion strains, and the ovine abortion strains were more closely related to the avian strains than the feline strains were. The results of a molecular analysis of the nucleotide sequences of the ompA gene (23) were consistent with the results of the DNA-DNA homology analyses. Our previous report indicated that restriction fragment length polymorphism (RFLP) of the rRNA operon region is a useful marker for typing and species identification (8).

Recently, the classification of bacterial species has been reevaluated by using genetic methods, including DNA-DNA homology, rRNA-DNA homology, and comparisons of 5S, 16S and 23S rRNA sequences, as well as other biochemical methods, including multilocus enzyme electrophoresis and fatty acid composition analysis $(24,29-31,36)$. Among the molecular analysis methods, rRNA or ribosomal DNA (rDNA) sequence comparisons provide a useful approach for studying phylogeny, especially the phylogeny of microorganisms with similar phenotypes, like the chlamydiae. rRNA gene sequences of avian C. psittaci (34), C. trachomatis (34), and C. pneumoniae $(9,10$, $34)$ strains have been reported. However, the sequences of $C$. pecorum and most of the mammal-derived $C$. psittaci strains have not been analyzed previously.

In this paper, we describe an analysis of the 16S rRNA gene sequences of $C$. psittaci, C. pecorum, and C. trachomatis strains. We also reviewed the sequences of $C$. pneumoniae and $C$. trachomatis to investigate the phylogenic relationships of these organisms in the genus Chlamydia. Our results indicated that the members of the genus Chlamydia can be separated into eight genetically distinguishable groups which form two genetic clusters.

\section{MATERIALS AND METHODS}

Chlamydial strains and DNAs. The chlamydial strains used in this study were 14 strains of $C$. psittaci, five strains of $C$. pecorum, and nine strains of $C$. tracho matis (Table 1). The procedures used for preparation of chlamydial DNA have been described previously (4). Koala type II DNA was kindly provided by N Itoh, Yokohama City University, Yokohama, Japan. C. trachomatis $\mathrm{A} / \mathrm{Har}-13^{\mathrm{T}}$ was kindly provided by C.-C. Kuo, University of Washington, Seattle. 
TABLE 1. Chlamydial strains investigated

\begin{tabular}{|c|c|c|c|c|c|c|}
\hline Species & Strain & Host & $\begin{array}{l}\text { Geographical } \\
\text { location }\end{array}$ & Disease & $\begin{array}{c}\text { RFLP } \\
\text { type }^{a}\end{array}$ & $\begin{array}{l}\text { Nucleotide sequence } \\
\text { accession no. }\end{array}$ \\
\hline \multirow[t]{17}{*}{ C. psittaci } & Prt/GCP-1 & Parrot & Japan & Systemic infection & Av1a & D85713 \\
\hline & Frt-Hu/Callo & Ferret, human & United States & Meningopneumonitis & $A v 1 b$ & D85712 \\
\hline & $\operatorname{Prk} / 6 \mathrm{BC} C^{\mathrm{T}}\left(=\mathrm{ATCC}\right.$ VR $\left.125^{\mathrm{T}}\right)$ & Parakeet & United States & Systemic infection & Av1c & $\mathrm{M} 13769^{b}$ \\
\hline & $\mathrm{Hu} / \mathrm{Borg}(=\mathrm{ATCC}$ VR601) & Human & United States & Psittacosis & Av1c & D85711 \\
\hline & Prk/Daruma & Parakeet & Japan (India) & Systemic infection & $\operatorname{Av} 2 \mathrm{a}$ & D85710 \\
\hline & Ov/B577 (= ATCC VR656) & Sheep & United States & Abortion & $A v 2 b$ & D85709 \\
\hline & EBA & Cattle & United States & Abortion & & U73108 \\
\hline & CPEAE & Sheep & United Kingdom & Abortion & & $\mathrm{Z} 49871^{b}$ \\
\hline & CPOAE & Sheep & United Kingdom & Abortion & & $\mathrm{Z} 49872^{b}$ \\
\hline & Gp/Ic (= ATCC VR813) & Guinea pig & United States & Conjunctivitis & Gp1 & D85708 \\
\hline & $\mathrm{Fe} / \mathrm{Pn}-1$ (= ATCC VR120) & Cat & United States & Pneumonia & Fela & D85701 \\
\hline & $\mathrm{Fe} / 145$ & Cat & United States & Conjunctivitis & Fela & D85702 \\
\hline & $\mathrm{Fe} /$ Cello & Cat & United States & Conjunctivitis & Fe1b & D85706 \\
\hline & $\mathrm{Fe} / \mathrm{B} 166$ & Cat & United Kingdom & Conjunctivitis & $\mathrm{Fe} 1 \mathrm{~b}$ & D85703 \\
\hline & $\mathrm{Fe} / \mathrm{C} 164$ & Cat & United Kingdom & Conjunctivitis & $\mathrm{Fe} 1 \mathrm{~b}$ & D85704 \\
\hline & $\mathrm{Fe} / \mathrm{C} 429$ & Cat & United Kingdom & Conjunctivitis & $\mathrm{Fe} 1 \mathrm{~b}$ & D85707 \\
\hline & $\mathrm{Fe} / \mathrm{C} 454$ & Cat & United Kingdom & Conjunctivitis & Fe1b & D85705 \\
\hline \multirow{5}{*}{ C. pecorum } & $\mathrm{Bo} / \mathrm{E} 58^{\mathrm{T}}\left(=\mathrm{ATCC}\right.$ VR628 $\left.{ }^{\mathrm{T}}\right)$ & Cattle & United States & Encephalomyelitis & Ru1a & D88317 \\
\hline & Bo/Maeda & Cattle & Japan & Pneumonia & Ru1a & D85715 \\
\hline & Bo/Shizuoka & Cattle & Japan & Encephalomyelitis & Ru1a & D85714 \\
\hline & Ov/IPA (= ATCC VR629) & Sheep & United States & Polyarthritis & $\mathrm{Ru} 1 \mathrm{~b}$ & D85716 \\
\hline & Koala (type II) & Koala & Australia & Urogenital infection & & D85717 \\
\hline \multirow{2}{*}{ C. pneumoniae } & TW183 $3^{\mathrm{T}}$ & Human & Taiwan & Conjunctivitis & & $\mathrm{L}_{0} 6108^{b}$ \\
\hline & IOL207 & Human & United Kingdom & Pneumonia & & $\mathrm{Z} 49874^{b}$ \\
\hline \multirow[t]{11}{*}{ C. trachomatis } & $\mathrm{A} / \mathrm{Har}-13^{\mathrm{T}}$ & Human & Egypt & Trachoma & & D89067 \\
\hline & A/SA-1/OT & Human & South Africa & Trachoma & & D88316 \\
\hline & B/TW-5/OT & Human & Taiwan & Trachoma & & D85719 \\
\hline & $\mathrm{C} / \mathrm{TW}-3 / \mathrm{OT}$ & Human & Taiwan & Trachoma & & D85720 \\
\hline & D/UW-3/Cx & Human & United States & Cervitis & & D85721 \\
\hline & $\mathrm{E} / \mathrm{UW}-5 / \mathrm{Cx}$ & Human & United States & Cervitis & $\mathrm{CtHu}$ & D85722 \\
\hline & $\mathrm{L} 2 / 434 / \mathrm{Bu}$ & Human & United States & Lymphogranuloma venereum & $\mathrm{CtHu}$ & M59178 \\
\hline & $\mathrm{R} 22$ & Pig & United States & Conjunctivitis & & U73109 \\
\hline & $\mathrm{S} 45$ & Pig & Austria & Asymptomatic & & U73110 \\
\hline & MoPn/Weiss-Nigg & Mouse & United States & Pneumonia & $\mathrm{CtMo}$ & D85718 \\
\hline & SFPD & Hamster & United States & Proliferative ileitis & & M83313 ${ }^{b}$ \\
\hline
\end{tabular}

${ }^{a}$ RFLP types were determined previously (5).

${ }^{b}$ Previously published $16 \mathrm{~S}$ rRNA sequence.

PCR amplification of chlamydial 16S rRNA gene fragments. Chlamydial 16S rRNA gene fragments were amplified by using a pair of primers (Table 2) constructed from the 16S rRNA gene sequence of $C$. psittaci $\operatorname{Prk} / 6 \mathrm{BC}^{\mathrm{T}}(\mathrm{T}=$ type strain) (34). These primers contain restriction sites for BamHI and SalI, which is convenient for further cloning. Amplification was performed in $100-\mu l$ mixtures which contained 1 to $5 \mathrm{ng}$ of genomic DNA and each primer at a concentration of $0.2 \mu \mathrm{M}$ in $10 \mathrm{mM}$ Tris- $\mathrm{HCl}$ buffer ( $\mathrm{pH} 8.3$ ) containing $50 \mathrm{mM} \mathrm{KCl}, 1.5 \mathrm{mM}$

TABLE 2. Primers used for rDNA amplification and direct sequencing

\begin{tabular}{|c|c|c|}
\hline Primer & Positions $^{a}$ & Sequence \\
\hline $16 \mathrm{~S} 1$ & -2 to 18 & 5'-CGGATCCTGAGAATTTGATC-3' \\
\hline $16 \mathrm{~S} 2$ & 1554 to 1537 & 5'-TGTCGACAAAGGAGGTGATCCA-3' \\
\hline rRNA1a & -1 to 21 & 5'-GGATCCTGAGAATTTGATCTTG-3' \\
\hline rRNA1b & 547 to 523 & 5'-TCCGTATTACCGCAGCTGCTGGCAC-3' \\
\hline rRNA01 & 523 to 547 & 5'-GTGCCAGCAGCTGCGGTAATACGGA-3' \\
\hline rRNA02 & 742 to 718 & 5'-GCCTTCGCCACTGGTGTTCTTCCAC-3' \\
\hline rRNA03 & 886 to 912 & 5'-TATGCCGCCTGAGGAGTACACTCGC-3' \\
\hline rRNA04 & 1076 to 1052 & 5'-TGACGACAGCCATGCAGCACCTGTG-3' \\
\hline rRNA4b & 1077 to 1099 & 5'-GCTCGTGCCGTGAGGTGTTGGGTTA-3' \\
\hline rRNA4a & 1554 to 1534 & 5'-TGTCGACAAAGGAGGTGATCCAGCC-3' \\
\hline
\end{tabular}

${ }^{a}$ The positions of nucleotides were determined by using an alignment of the 16S rRNA gene sequences of 35 strains of chlamydiae.
$\mathrm{MgCl}_{2}$, and each deoxynucleoside triphosphate at a concentration of $200 \mu \mathrm{M}$ The reactants were overlaid with $50 \mu \mathrm{l}$ of mineral oil and subjected to thermal cycle amplification. The program consisted of initial melting at $94^{\circ} \mathrm{C}$ for $3 \mathrm{~min}$, four cycles of denaturation at $94^{\circ} \mathrm{C}$ for $1 \mathrm{~min}$, annealing at $40^{\circ} \mathrm{C}$ for $1 \mathrm{~min}$, and extension at $72^{\circ} \mathrm{C}$ for $2 \mathrm{~min}, 36$ cycles of denaturation at $94^{\circ} \mathrm{C}$ for $1 \mathrm{~min}$, annealing at $50^{\circ} \mathrm{C}$ for $1 \mathrm{~min}$, and extension at $72^{\circ} \mathrm{C}$ for $2 \mathrm{~min}$, and a final extension step at $72^{\circ} \mathrm{C}$ for $10 \mathrm{~min}$, followed by soaking at $4^{\circ} \mathrm{C}$; a model 9600 GeneAmp PCR System thermal cycler (Perkin-Elmer Cetus, ABI, Tokyo, Japan) was used. Amplified products were purified by agarose gel electrophoresis. DNA was extracted from agarose gels by using a GeneClean II kit (Bio 101, Inc., La Jolla, Calif.) as recommended by the manufacturer.

Sequencing of rDNA. The $16 \mathrm{~S}$ rRNA gene fragment was inserted into pTV119N between the SalI and BamHI sites and then transfected into Escherichia coli MV1184. Recombinant plasmids were extracted and purified by the alkaline method (3). Direct sequences were also examined by using purified PCR products. Nucleotide sequences were determined by the dideoxy chain termination method (28). All sequencing was performed with AutoRead and AutoCycle sequencing kits and an ALFred sequencer (model 373A automated DNA sequencing system; Pharmacia, Tokyo, Japan). The sequencing primers used were -40 forward and reverse primers for pTV119N sites and internal primers for sites within the 16S rRNA gene (Table 2). Sequences were determined for both strands at least twice and were edited with the GCG Wisconsin computer program package (1).

Sequence analysis. In addition to the $16 \mathrm{~S}$ rRNA gene sequences determined in this study, previously published sequences were also analyzed. These included the sequences of $C$. psittaci Prk/6BC ${ }^{\mathrm{T}}$ (accession no. M13769), CPEAE (accession no. Z49871), and CPOAE (accession no. Z49872), C. pneumoniae TW183 (accession no. L06108) and IOL207 (accession no. Z49874), C. trachomatis SFPD (accession no. M83313) and L2/434/Bu (accession no. M59178), and the 


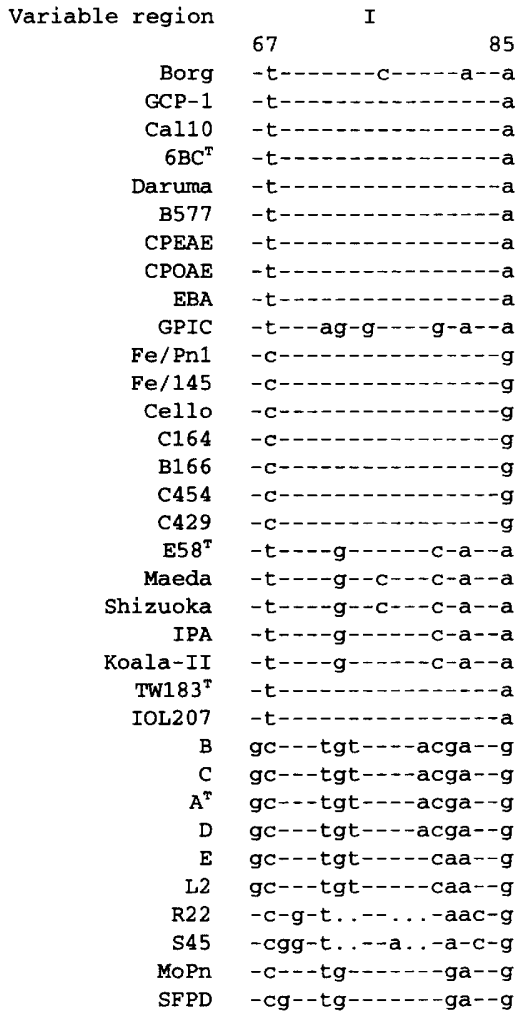

Consensus A AATGACTTCGCTTCTT-

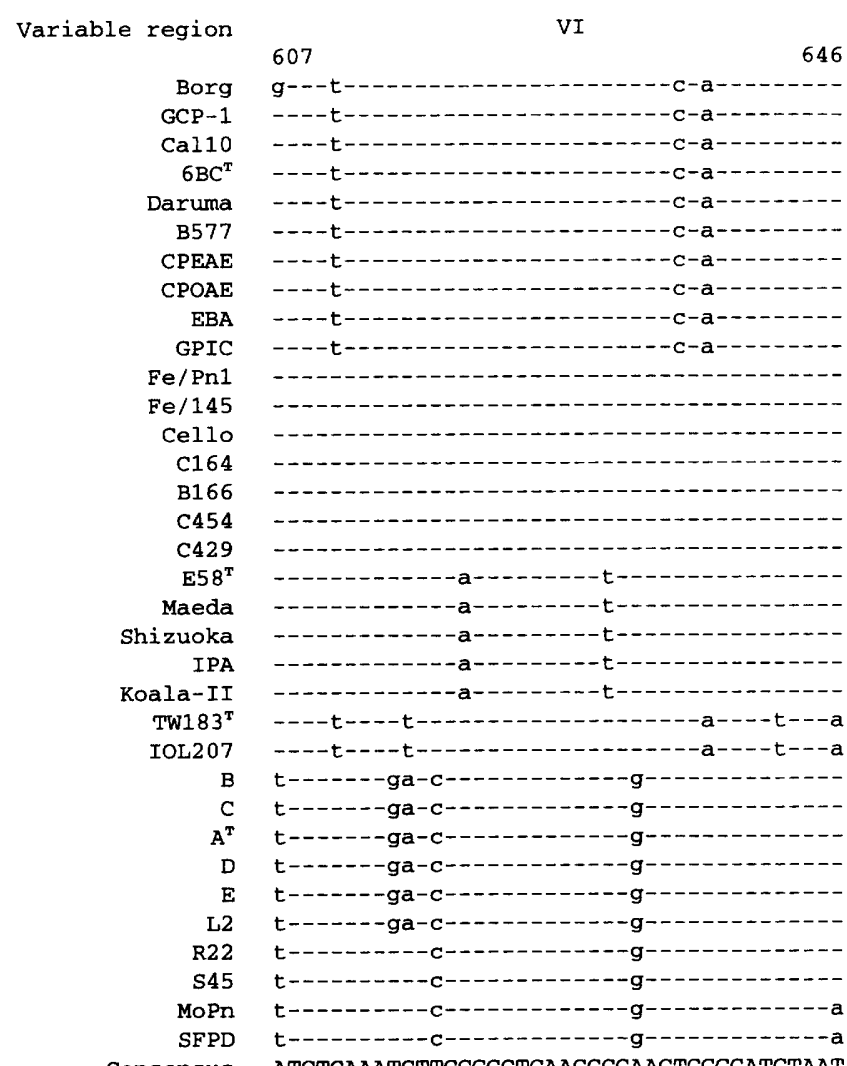

III

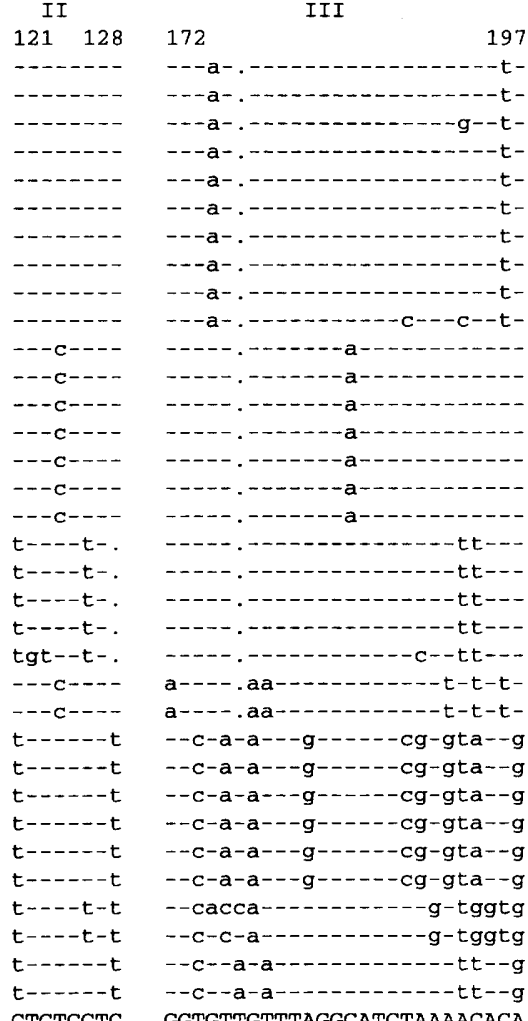

CTGTCCTC GGTGTOTTAGCATCTAAAACACA

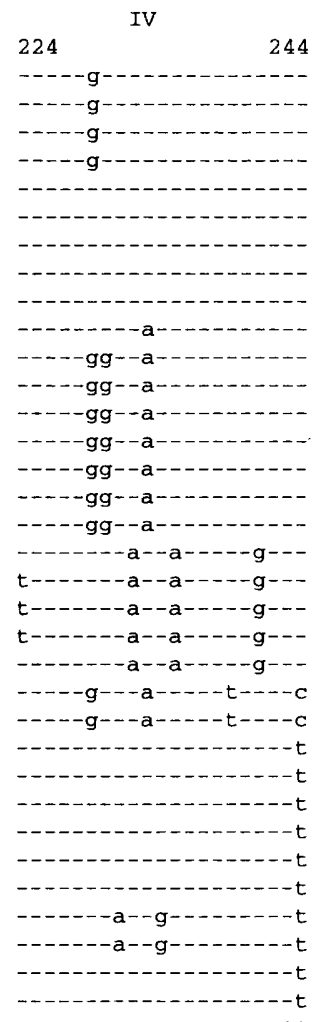

CGGTTAAGGGAGAGTCTATGG

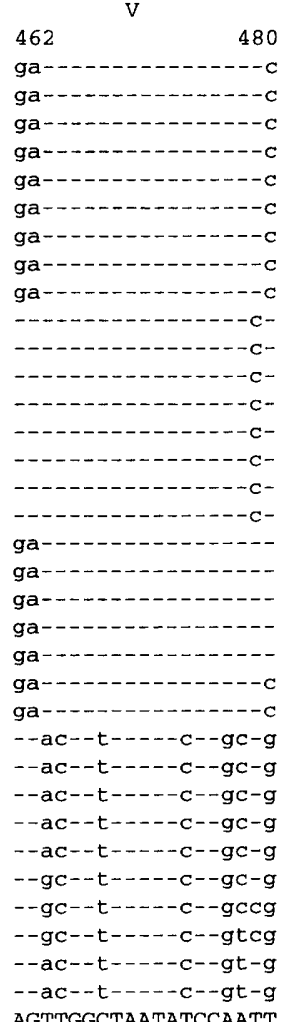

AGTTGGCTAATATCCAATT

1005

VII

VIII

$1047 \quad 145$ 1467

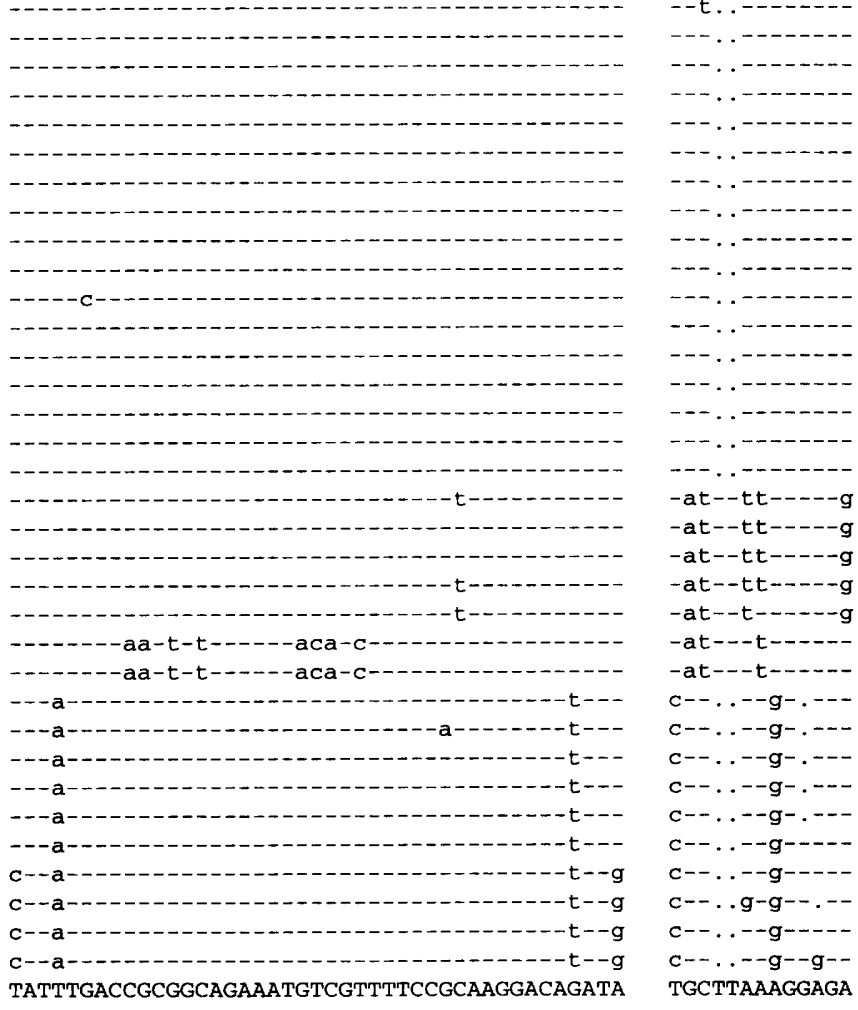

FIG 1. Comparison of the variable regions of the 16S rRNA gene sequences of $C$. psittaci, $C$. pecorum, $C$. pneumoniae, and $C$. trachomatis. Consensus sequences are shown at the bottom. Nucleotides that are different from the nucleotides in the consensus sequence are in lowercase letters. Dots indicate deletions. 
TABLE 3. Dissimilarities and genetic distances in rDNA sequences of Chlamydia spp.

\begin{tabular}{|c|c|c|c|c|c|c|c|c|c|c|c|c|c|c|c|c|c|}
\hline \multirow{2}{*}{ Strain } & \multicolumn{17}{|c|}{ Dissimilarity or genetic distance ${ }^{a}$} \\
\hline & $\begin{array}{l}\mathrm{Hu} / \\
\text { Borg }\end{array}$ & $\begin{array}{c}\text { Prt/ } \\
\text { GCP-1 }\end{array}$ & $\begin{array}{c}\text { Frt-Hu/ } \\
\text { Cal10 }\end{array}$ & $\begin{array}{l}\text { Prk/ } \\
6 \mathrm{BC}^{\mathrm{T}}\end{array}$ & $\begin{array}{c}\text { Prk/ } \\
\text { Daruma }\end{array}$ & $\begin{array}{l}\text { Ov/ } \\
\text { B577 }\end{array}$ & CPEAE & CPOAE & EBA & $\mathrm{Gp} / \mathrm{Ic}$ & $\begin{array}{l}\mathrm{Fe} / \\
\mathrm{Pn}-1\end{array}$ & $\begin{array}{l}\mathrm{Fe} / \\
145\end{array}$ & $\begin{array}{c}\mathrm{Fe} / \\
\text { Cello }\end{array}$ & $\begin{array}{c}\mathrm{Fe} / \\
\mathrm{C} 164\end{array}$ & $\begin{array}{c}\mathrm{Fe} / \\
\mathrm{B} 166\end{array}$ & $\begin{array}{c}\mathrm{Fe} / \\
\mathrm{C} 454\end{array}$ & $\begin{array}{c}\mathrm{Fe} / \\
\mathrm{C} 429\end{array}$ \\
\hline $\mathrm{Hu} /$ Borg & 0 & 0.58 & 0.52 & 0.39 & 0.71 & 0.78 & 0.75 & 0.75 & 0.71 & 1.36 & 2.00 & 1.68 & 1.74 & 1.81 & 1.81 & 1.94 & 1.81 \\
\hline $\mathrm{Prt} / \mathrm{GCP}-1$ & 0.0058 & 0 & 0.32 & 0.19 & 0.52 & 0.58 & 0.55 & 0.55 & 0.52 & 1.29 & 1.81 & 1.49 & 1.55 & 1.61 & 1.61 & 1.74 & 1.55 \\
\hline Frt-Hu/Cal10 & 0.0052 & 0.0032 & 0 & 0.13 & 0.45 & 0.52 & 0.48 & 0.48 & 0.45 & $\begin{array}{l}1.16 \\
1.16\end{array}$ & 1.74 & 1.42 & 1.49 & 1.55 & 1.55 & 1.68 & 1.55 \\
\hline $\operatorname{Prk} / 6 \mathrm{BC}^{\mathrm{T}}$ & 0.0039 & 0.0019 & 0.0013 & 0 & 0.32 & 0.39 & 0.34 & 0.34 & 0.32 & $\begin{array}{l}1.1 \\
\end{array}$ & 1.61 & 1.29 & 1.36 & 1.42 & 1.42 & 1.55 & 1.42 \\
\hline Prk/Daruma & 0.0071 & 0.0052 & 0.0045 & 0.0032 & 0 & 0.32 & 0.27 & 0.27 & 0.26 & $\begin{array}{l}1.03 \\
\end{array}$ & 1.94 & 1.61 & 1.68 & 1.74 & 1.74 & 1.87 & 1.74 \\
\hline $\mathrm{Ov} / \mathrm{B} 577$ & 0.0078 & 0.0058 & 0.0052 & 0.0039 & 0.0032 & 0 & 0.07 & 0.07 & 0.06 & 1.10 & 2.00 & 1.68 & 1.74 & 1.81 & 1.81 & 1.94 & 1.81 \\
\hline CPEAE & 0.0075 & 0.0055 & 0.0048 & 0.0034 & 0.0027 & 0.0007 & 0 & 0 & 0 & $\begin{array}{l}1.09 \\
\end{array}$ & 2.05 & 1.71 & 1.77 & 1.84 & 1.84 & 1.98 & 1.84 \\
\hline CPOAE & 0.0075 & 0.0055 & 0.0048 & 0.0034 & 0.0027 & 0.0007 & 0.0000 & 0 & 0 & 1.09 & 2.05 & 1.71 & 1.77 & 1.84 & 1.84 & 1.98 & 1.84 \\
\hline EBA & 0.0071 & 0.0052 & 0.0045 & 0.0032 & 0.0026 & 0.0006 & 0.0000 & 0.0000 & 0 & 1.03 & 1.94 & 1.61 & 1.68 & 1.74 & 1.74 & 1.87 & 1.74 \\
\hline $\mathrm{Gp} / \mathrm{Ic}$ & 0.0137 & 0.0130 & 0.0117 & 0.0111 & 0.0104 & 0.0111 & 0.0110 & & & 0 & 2.07 & 1.74 & 1.81 & 1.87 & 1.87 & 2.00 & 1.87 \\
\hline $\mathrm{Fe} / \mathrm{Pn}-1$ & 0.0203 & 0.0183 & 0.0176 & 0.0163 & 0.0196 & 0.0203 & 0.0207 & 0.0207 & 0.0196 & 0.0210 & 0 & 0.32 & 0.39 & 0.32 & 0.45 & 0.52 & 0.45 \\
\hline $\mathrm{Fe} / 145$ & 0.017 & 0.0150 & 0.0143 & 0.0130 & 0.0163 & 0.0170 & 0.0173 & 0.0173 & 0.0163 & 0.0176 & 0.0032 & 0 & 0.06 & 0.13 & 0.13 & 0.26 & 0.13 \\
\hline $\mathrm{Fe} /$ Cello & 0.0176 & 0.0157 & 0.0150 & 0.0137 & 0.0170 & 0.0176 & 0.0179 & 0.0179 & 0.0170 & 0.0183 & 0.0039 & 0.0006 & 0 & 0.06 & 0.19 & 0.19 & 0.06 \\
\hline $\mathrm{Fe} / \mathrm{C} 164$ & 0.0183 & 0.0163 & 0.0157 & 0.0143 & 0.0176 & 0.0183 & 0.0186 & $0 .($ & 0.0 & 0.0 & $\begin{array}{l}0.0 \\
0.0\end{array}$ & 0.0013 & 0.0006 & 0 & 0.26 & 0.19 & 0.13 \\
\hline $\mathrm{Fe} / \mathrm{B} 166$ & 0.0183 & 0.0163 & 0.0157 & 0.0143 & 0.0176 & 0.0183 & 0.0186 & 0.0186 & 0.0176 & 0.0190 & 0.0045 & 0.0013 & 0.0019 & 0.0026 & 0 & 0.39 & 0.26 \\
\hline $\mathrm{Fe} / \mathrm{C} 454$ & 0.0196 & 0.0176 & 0.0170 & 0.0157 & 0.0190 & 0.0196 & 0.0200 & 0.0200 & 0.0190 & 0.0203 & 0.0052 & 0.0026 & 0.0019 & 0.0019 & 0.0039 & 0 & 0.26 \\
\hline $\mathrm{Fe} / \mathrm{C} 429$ & 0.0183 & 0.0157 & 0.0157 & 0.0143 & 0.0176 & 0.0183 & 0.0186 & 0.0186 & 0.0176 & 0.0190 & 0.0045 & 0.0013 & . & .0 .0013 & 0.0026 & 0.0026 & 0 \\
\hline $\mathrm{Bo} / \mathrm{E} 58^{\mathrm{T}}$ & 364 & 0.0344 & 0.0358 & 0351 & 0371 & 0378 & 0.0393 & 0.0393 & 0.0371 & 0.0392 & 0.0426 & 0.0392 & 0.0 & 0.0405 & 0.0405 & 0.0419 & 0.0 \\
\hline Bo/Maeda & 0.0371 & 0.0364 & 0.0378 & 0.0371 & 0.0392 & 0.0399 & 0.0414 & 0.0414 & 0.0392 & 0.0412 & 0.0446 & 0.0412 & 0.0419 & 0.0426 & 0.0426 & 0.0440 & 0.0426 \\
\hline Bo/Shizuoka & 0371 & 0.0364 & 0.0378 & 0.0371 & 0.0392 & 0.0399 & 0.0414 & 0.0414 & 0.0392 & 0.0412 & 0.0446 & 0.0412 & 0.0419 & 0.0426 & 0.0426 & 0.0440 & 0.0426 \\
\hline Ov/IPA & 0.0371 & 0.0351 & 0.0364 & 0.0358 & 0.0378 & 0.0385 & 0.0400 & 0.0400 & 0.0378 & 0.0399 & 0.0433 & 0.0399 & 0.0405 & 0.0412 & 0.0412 & 0.0426 & 0.0412 \\
\hline Koala-II & 0.0371 & 0.0351 & 0.0364 & 0.0358 & 0.0378 & 0.0385 & 0.0400 & 0.0400 & 0.0378 & 0.0399 & 0.0433 & 0.0399 & 0.0405 & 0.0412 & 0.0412 & 0.0426 & 0.0412 \\
\hline TW183 ${ }^{\mathrm{T}}$ & 412 & 384 & 405 & 391 & 25 & 2 & 0.0443 & 43 & 25 & & 08 & 73 & & & & & \\
\hline IOL207 & 0.0412 & 0.0384 & 0.0405 & 0.0391 & 0.0425 & 0.0432 & 0.0443 & 0.0443 & 0.0425 & 0.0487 & 0.0508 & 0.0473 & 0.0480 & 0.0487 & 0.0487 & 0.0501 & 0.0487 \\
\hline $\mathrm{B} / \mathrm{TW}-5 / \mathrm{OT}$ & 0522 & & & & & & & & & & & & & & & & \\
\hline C/TW-3/OT & 0.0522 & 0.0529 & 0.0515 & 0.0508 & 0.0515 & 0.0522 & 0.0538 & 0.0538 & 0.0515 & 0.0494 & 0.0550 & 0.0515 & 0.0522 & 0.0529 & 0.0529 & 0.0543 & 0.0529 \\
\hline $\mathrm{A} / \mathrm{Har}-13^{\mathrm{T}}$ & 0.0515 & 0.0522 & 0.0508 & 0.0501 & 0.0508 & 0.0515 & 0.0530 & 0.0530 & 0.0508 & 0.0487 & 0.0543 & 0.0508 & 0.0515 & 0.0522 & 0.0522 & 0.0536 & 0.0522 \\
\hline $\mathrm{D} / \mathrm{UW}-3 / \mathrm{Cx}$ & 0.0515 & 0.0522 & 0.0508 & 0.0501 & 0.0508 & 0.0515 & 0.0530 & 0.0530 & 0.0508 & 0.0487 & 0.0543 & 0.0508 & 0.0515 & 0.0522 & 0.0522 & 0.0536 & 0.0522 \\
\hline E/UW-5/Cx & 0.0501 & 0.0508 & 0.0494 & 0.0487 & 0.0494 & 0.0515 & 0.0530 & 0.0530 & 0.0508 & 0.0487 & 0.0529 & 0.0494 & 0.0501 & 0.0508 & 0.0494 & 0.0522 & 0.0508 \\
\hline $\mathrm{L} 2 / 434 / \mathrm{Bu}$ & 0.0494 & 0.0501 & 0.0487 & 0.0480 & 0.0501 & 0.0508 & 0.0515 & 0.0515 & 0.0501 & 0.0480 & 0.0522 & 0.0487 & 0.0494 & 0.0501 & 0.0501 & 0.0515 & 0.0501 \\
\hline $\mathrm{R} 22$ & 0.0510 & 0.0517 & 0.0503 & 0.0496 & 0.0517 & 0.0524 & 0.0539 & 0.0539 & 0.0517 & 0.0510 & 0.0538 & 0.0503 & 0.0510 & 0.0517 & 0.0517 & 0.0531 & 0.0517 \\
\hline $\mathrm{S} 45$ & 0.0577 & 0.0570 & 0.0556 & 0.0549 & 0.0570 & 0.0577 & 0.0591 & 0.0591 & 0.0570 & 0.0577 & 0.0577 & 0.0542 & 0.0549 & 0.0556 & 0.0556 & 0.0570 & 0.0556 \\
\hline $\begin{array}{l}\text { MoPn/Weiss- } \\
\text { Nigg }\end{array}$ & 0.0446 & 0.0453 & 0.0439 & 0.0432 & 0.0439 & 0.0446 & 0.0464 & 0.0464 & 0.0439 & 0.0439 & 0.0460 & 0.0425 & 0.0432 & 0.0439 & 0.0439 & 0.0453 & 0.0439 \\
\hline SFPD & 0.0463 & 0.0471 & 0.0456 & 0.0449 & 0.0456 & 0.0463 & 0.0464 & 0.0464 & 0.0456 & 0.0456 & 0.0485 & 0.0449 & 0.0456 & 0.0463 & 0.0463 & 0.0478 & 0.0463 \\
\hline "Z" & 0.1773 & 0.1765 & 0.1756 & 0.1748 & 0.1765 & 0.1773 & 0.1829 & 0.1829 & 0.1765 & 0.1724 & 0.1756 & 0.1716 & 0.1724 & 0.1732 & 0.1732 & 0.1748 & 0.1732 \\
\hline
\end{tabular}

${ }^{a}$ The values on the upper right are numbers of substitutions per 100 bases. The values on the lower left are the genetic distances calculated by the Jukes-Cantor method. The values inside each solid box are the values for a species. The values inside the dotted boxes are sequence dissimilarity values of less than $1 \%$ and genetic distances of less than 0.01 . The rDNA sequence of strain SFPD contains 19 ambiguous sites, which were not included in the analysis.

chlamydia-like microorganism Simkania sp. strain "Z" (accession no. L27666); these sequences were obtained from the DNA Data Bank of Japan (DDBJ), Mishima, Japan. The 16S rRNA gene sequences of $C$. psittaci EBA (accession no. U73108) and C. trachomatis R22 (accession no. U73109) and S45 (accession no. U73110) were kindly provided by Karin D. E. Everett and Arthur A. Andersen of the Avian and Swine Respiratory Diseases Research Unit, USDA Agricultural Research Service National Animal Disease Center, Ames, Iowa. Multiple alignments of the 16S rRNA gene sequences were examined by using the computer program ClustalW (17) and a SUN workstation. Genetic distances were calculated by the method of Jukes and Cantor (21). A phylogenetic tree was constructed by the neighbor-joining method (27). The molecular phylogenetic analysis results were analyzed by using the program package ODEN (20) at the National Institute of Genetics, Mishima, Japan, the PHYLIP package (2), and the GCG Wisconsin package (1) on the SUN workstation at Gifu University Computing Center, Gifu, Japan.

Nucleotide sequence accession number. The nucleotide sequence data re ported in this paper have been deposited in the DDBJ, EMBL, and GenBank nucleotide sequence databases under the accession numbers listed in Table 1.

\section{RESULTS}

The 16S rRNA genes of the Chlamydia strains analyzed were amplified with primers 16S1 and 16S2. The following two strategies were employed to determine the sequences: (i) cloningsubcloning and sequencing and (ii) direct sequencing. The cloning-subcloning method was initially used for the PCR products from $C$. psittaci Prt/GCP-1, Prk/Daruma, and Fe/Pn-1 and C. pecorum $\mathrm{Bo} / \mathrm{Maeda}$ and Bo/Shizuoka. Based on these sequences and the previously reported sequences, internal direct sequencing primers were designed for the chlamydial $16 \mathrm{~S}$ rRNA gene (Table 2) for subsequent direct sequencing. In order to confirm the fidelity of the direct sequencing, the sequence of the $16 \mathrm{~S}$ rRNA gene of Prk $/ 6 \mathrm{BC}^{\mathrm{T}}$ was determined for comparison with the previously reported sequence (34). Only one nucleotide mismatch between the two sequences was found. This mismatch was reproducible in several repeated sequence analyses. Therefore, the mismatch was not caused by a sequencing error. Thus, the reliability of the sequences determined was greater than $99.94 \%$.

The 16S rRNA gene sequences of 27 chlamydial strains were determined. In addition, seven sequences from DNA databases were included in the analysis. The $16 \mathrm{~S}$ rRNA gene sequences were composed of $1,548 \mathrm{bp}$ in both $C$. psittaci and human $C$. trachomatis strains, 1,544 bp in swine $C$. trachomatis strains, 1,549 bp in both rodent $C$. trachomatis and C. pecorum strains, and 1,550 bp in $C$. pneumoniae strains. The consensus 
TABLE 3-Continued

\begin{tabular}{|c|c|c|c|c|c|c|c|c|c|c|c|c|c|c|c|c|c|}
\hline \multicolumn{18}{|c|}{ Dissimilarity or genetic distance ${ }^{a}$} \\
\hline $\begin{array}{l}\mathrm{Bo} / \\
\mathrm{E} 58^{\mathrm{T}}\end{array}$ & $\begin{array}{c}\text { Bo/ } \\
\text { Maeda }\end{array}$ & $\begin{array}{c}\mathrm{Bo} / \\
\text { Shizuoka }\end{array}$ & $\begin{array}{l}\text { Ov/ } \\
\text { IPA }\end{array}$ & $\begin{array}{c}\text { Koala } \\
\text { II }\end{array}$ & $\mathrm{TW} 183^{\mathrm{T}}$ & IOL207 & $\begin{array}{c}\text { B/ } \\
\text { TW-5/ } \\
\text { OT }\end{array}$ & $\begin{array}{c}\mathrm{C} / \\
\text { TW-3/ } \\
\text { OT }\end{array}$ & $\begin{array}{c}\mathrm{A} / \\
\text { Har-13 }\end{array}$ & $\begin{array}{c}\text { D/ } \\
\text { UW-3/ } \\
\text { Cx }\end{array}$ & $\begin{array}{c}\text { E/ } \\
\text { UW-5/ } \\
\text { Cx }\end{array}$ & $\begin{array}{c}\mathrm{L} 2 / \\
434 / \\
\mathrm{Bu}\end{array}$ & $\mathrm{R} 22$ & S45 & $\begin{array}{c}\text { MoPn/ } \\
\text { Weiss- } \\
\text { Nigg }\end{array}$ & SFPD & “Z” \\
\hline 3.56 & 3.62 & 3.62 & 3.62 & 3.62 & 4.01 & 4.01 & 5.04 & 5.04 & 4.98 & 4.98 & 4.85 & 4.78 & 4.93 & 5.56 & 4.33 & 5.71 & 15.8 \\
\hline 3.36 & 3.56 & 3.56 & 3.43 & 3.43 & 3.75 & 3.75 & 5.11 & 5.11 & 5.04 & 5.04 & 4.91 & 4.84 & 4.99 & 5.49 & 4.39 & 5.78 & 15.7 \\
\hline 3.49 & 3.69 & 3.69 & 3.56 & 3.56 & 3.94 & 3.94 & 4.98 & 4.98 & 4.91 & 4.91 & 4.78 & 4.72 & 4.86 & 5.36 & 4.26 & 5.65 & 15.7 \\
\hline 3.43 & 3.62 & 3.62 & 3.49 & 3.49 & 3.81 & 3.81 & 4.91 & 4.91 & 4.85 & 4.85 & 4.72 & 4.65 & 4.8 & 5.29 & 4.2 & 5.58 & 15.6 \\
\hline 3.62 & 3.82 & 3.82 & 3.69 & 3.69 & 4.13 & 4.13 & 4.98 & 4.98 & 4.91 & 4.91 & 4.78 & 4.84 & 4.99 & 5.49 & 4.26 & 5.65 & 15.7 \\
\hline 3.69 & 3.88 & 3.88 & 3.75 & 3.75 & 4.20 & 4.20 & 5.04 & 5.04 & 4.98 & 4.98 & 4.98 & 4.91 & 5.06 & 5.56 & 4.33 & 5.71 & 15.8 \\
\hline 3.83 & 4.03 & 4.03 & 3.89 & 3.89 & 4.30 & 4.30 & 5.19 & 5.19 & 5.12 & 5.12 & 5.12 & 4.98 & 5.20 & 5.68 & 4.50 & 5.74 & 16.2 \\
\hline 3.83 & 4.03 & 4.03 & 3.89 & 3.89 & 4.30 & 4.30 & 5.19 & 5.19 & 5.12 & 5.12 & 5.12 & 4.98 & 5.20 & 5.68 & 4.50 & 5.74 & 16.2 \\
\hline 3.62 & 3.82 & 3.82 & 3.69 & 3.69 & 4.13 & 4.13 & 4.98 & 4.98 & 4.91 & 4.91 & 4.91 & 4.84 & 4.99 & 5.49 & 4.26 & 5.65 & 15.7 \\
\hline 3.82 & 4.01 & 4.01 & 3.88 & 3.88 & 4.72 & 4.72 & 4.78 & 4.78 & 4.72 & 4.72 & 4.72 & 4.65 & 4.93 & 5.56 & 4.26 & 5.65 & 15.4 \\
\hline 4.14 & 4.33 & 4.33 & 4.20 & 4.20 & 4.91 & 4.91 & 5.30 & 5.30 & 5.24 & 5.24 & 5.11 & 5.04 & 5.19 & 5.56 & 4.46 & 5.91 & 15.7 \\
\hline 3.82 & 4.01 & 4.01 & 3.88 & 3.88 & 4.59 & 4.59 & 4.98 & 4.98 & 4.91 & 4.91 & 4.78 & 4.72 & 4.86 & 5.23 & 4.13 & 5.58 & 15.3 \\
\hline 3.88 & 4.08 & 4.08 & 3.95 & 3.95 & 4.65 & 4.65 & 5.04 & 5.04 & 4.98 & 4.98 & 4.85 & 4.78 & 4.93 & 5.29 & 4.20 & 5.65 & 15.4 \\
\hline 3.95 & 4.14 & 4.14 & 4.01 & 4.01 & 4.72 & 4.72 & 5.11 & 5.11 & 5.04 & 5.04 & 4.91 & 4.84 & 4.99 & 5.36 & 4.26 & 5.71 & 15.5 \\
\hline 3.95 & 4.14 & 4.14 & 4.01 & 4.01 & 4.72 & 4.72 & 4.98 & 5.11 & 5.04 & 5.04 & 4.78 & 4.84 & 4.99 & 5.36 & 4.26 & 5.71 & 15.5 \\
\hline 4.08 & 4.27 & 4.27 & 4.14 & 4.14 & 4.84 & 4.84 & 5.24 & 5.24 & 5.17 & 5.17 & 5.04 & 4.97 & 5.12 & 5.49 & 4.39 & 5.85 & 15.6 \\
\hline 3.95 & 4.14 & 4.14 & 4.01 & 4.01 & 4.72 & 4.72 & 5.11 & 5.11 & 5.04 & 5.04 & 4.91 & 4.84 & 4.99 & 5.36 & 4.26 & 5.71 & 15.5 \\
\hline 0 & 0.32 & 0.32 & 0.06 & 0.32 & 4.20 & 4.20 & 4.98 & 4.98 & 4.92 & 4.92 & 4.85 & 4.79 & 5.26 & 5.43 & 4. & 5.79 & 15.7 \\
\hline 0.0032 & 0 & 0.00 & 0.26 & 0.65 & 4.39 & 4.39 & 5.18 & 5.18 & 5.11 & 5.11 & 5.05 & 4.98 & 5.45 & 5.63 & 4.59 & 5.99 & 15.8 \\
\hline 0.0032 & 0.0000 & 0 & 0.26 & 0.65 & 4.39 & 4.39 & 5.18 & 5.18 & 5.11 & 5.11 & 5.05 & 4.98 & 5.45 & 5.63 & 4.59 & 5.99 & 15.8 \\
\hline 0.0006 & 0.0026 & 0.0026 & 0 & 0.39 & 4.26 & 4.26 & 5.05 & 5.05 & 4.98 & 4.98 & 4.92 & 4.85 & 5.32 & 5.5 & 4.46 & 5.85 & 15.8 \\
\hline 0.0032 & 0.0065 & 0.0065 & 0.0039 & 0 & 4.39 & 4.39 & 4.98 & 4.98 & 4.92 & 4.92 & 4.85 & 4.79 & 5.32 & 5.5 & 4.46 & 5.85 & 15.7 \\
\hline 0.0432 & 0.0453 & 0.0453 & 0.0439 & 0.0453 & $0^{\circ}$ & 0.000 & 6.14 & 6.14 & 6.08 & 6.08 & 6.08 & 6.01 & 6.49 & 6.47 & 5.49 & 6.92 & 15.9 \\
\hline 0.0432 & 0.0453 & 0.0453 & 0.0439 & 0.0453 & 0.0000 & & 6.14 & 6.14 & 6.08 & 6.08 & 6.08 & 6.01 & 6.49 & 6.47 & 5.49 & 6.92 & 15.9 \\
\hline 0.0516 & 0.0537 & 0.0537 & 0.0523 & 0.0516 & 0.0641 & 0.0641 & 0 & 0.13 & 0.06 & 0.06 & 0.39 & 0.52 & 2.59 & 2.68 & 1.68 & 3.02 & 15.8 \\
\hline 0.0516 & 0.0537 & 0.0537 & 0.0523 & 0.0516 & 0.0641 & 0.0641 & 0.0013 & 0 & 0.06 & 0.06 & 0.52 & 0.52 & 2.59 & 2.68 & 1.68 & 3.02 & 15.8 \\
\hline 0.0509 & 0.0530 & 0.0530 & 0.0516 & 0.0509 & 0.0634 & 0.0634 & 0.0006 & 0.0006 & 0 & 0 & 0.45 & 0.45 & 2.53 & 2.61 & 1.61 & 2.96 & 15.7 \\
\hline 0.0509 & 0.0530 & 0.0530 & 0.0516 & 0.0509 & 0.0634 & 0.0634 & 0.0006 & 0.0006 & 0.0000 & 0 & 0.45 & 0.45 & 2.53 & 2.61 & 1.61 & 2.96 & 15.7 \\
\hline 0.0502 & 0.0523 & 0.0523 & 0.0509 & 0.0502 & 0.0634 & 0.0634 & 0.0039 & 0.0052 & 0.0045 & 0.0045 & 0 & 0.26 & 2.4 & 2.48 & 1.68 & 3.02 & 15.7 \\
\hline 0.0495 & 0.0515 & 0.0515 & 0.0502 & 0.0495 & 0.0626 & 0.0626 & 0.0052 & 0.0052 & 0.0045 & 0.0045 & 0.0026 & 0 & 2.27 & 2.35 & 1.68 & 2.96 & 15.5 \\
\hline 0.0545 & 0.0566 & 0.0566 & 0.0552 & 0.0552 & 0.0678 & 0.0678 & 0.0264 & 0.0264 & 0.0257 & 0.0257 & 0.0244 & 0.0230 & 0 & 1.05 & 2.01 & 3.37 & 15.1 \\
\hline 0.0564 & 0.0585 & 0.0585 & 0.0571 & 0.0571 & 0.0677 & 0.0677 & 0.0273 & 0.0273 & 0.0266 & 0.0266 & 0.0253 & 0.0239 & 0.0105 & 0 & 2.16 & 3.33. & 15.4 \\
\hline 0.0453 & 0.0474 & 0.0474 & 0.0460 & 0.0460 & 0.0570 & 0.0570 & 0.0170 & 0.0170 & 0.0163 & 0.0163 & 0.0170 & 0.0170 & 0.0204 & 0.0219 & 0 & 1.54 & 15.5 \\
\hline 0.0471 & 0.0493 & 0.0493 & 0.0478 & 0.0478 & 0.0595 & 0.0595 & 0.0179 & 0.0179 & 0.0172 & 0.0172 & 0.0179 & 0.0172 & 0.0215 & 0.0210 & 0.0027 & $\ldots$ & 17.0 \\
\hline 0.1767 & 0.1776 & 0.1776 & 0.1776 & 0.1759 & 0.1781 & 0.1781 & 0.1773 & 0.1773 & 0.1765 & 0.1765 & 0.1756 & 0.1739 & 0.1685 & 0.1724 & 0.1731 & 0.1793 & 0 \\
\hline
\end{tabular}

sequence was constructed and assigned a number based on the multiple alignment. Nucleotide insertions or deletions were observed at 12 sites, including positions $73,74,77,78,79,128$, $178,753,1,458,1,459,1,464$, and 1,465 . Variations in the chlamydial 16S rRNA gene sequences were evident in eight areas (Fig. 1). These areas were designated variable regions I to VIII, which corresponded to nucleotides 67 to 85,121 to 128 , 172 to 197,224 to 244,462 to 480,607 to 646,1005 to 1047 , and 1455 to 1467 , respectively. These regions were mapped to the double helical stem or hairpin portions of the predicted secondary structure of the 16S rRNA molecule (16). The sequences in these variable regions exhibited genetic group specificity.

The sequences of the chlamydial 16S rRNA gene were highly conserved. The levels of sequence dissimilarity and the genetic distances were 0 to $6.21 \%$ and 0 to 0.0648 , respectively, among the chlamydiae (Table 3 ). The previously published sequences of $C$. pneumoniae TW183 ${ }^{\mathrm{T}}$ and IOL207, which were obtained from the DDBJ, were identical. The distribution of dissimilarities and genetic distances revealed three peaks. The levels of dissimilarity for these peaks ranged from 0 to $0.78 \%$, from 1.09 to $2.10 \%$, and from 3.43 to $6.21 \%$, and the genetic distances ranged from 0 to 0.0078 , from 0.0104 to 0.0210 , and from 0.0351 to 0.0648 . The levels of dissimilarity and genetic distances within species were 0 to $2.07 \%$ and 0 to 0.0210 , respectively, in $C$. psittaci, 0 to $0.65 \%$ and 0 to 0.0065 , respectively, in $C$. peconum, and 0 to $3.37 \%$ and 0 to 0.0273 , respectively in $C$. trachomatis. Thus, the $C$. psittaci and $C$. trachomatis sequence data revealed two peaks of dissimilarity and genetic distance within the species. Sequences that fell within the range of values for each peak were considered members of a group. A total of eight groups were identified. These groups consisted of (i) avian and ovine abortion strains of $C$. psittaci, (ii) a guinea pig strain of $C$. psittaci, (iii) feline strains of $C$. psittaci, (iv) C. pecorum, (v) C. pneumoniae, (vi) human strains of $C$. trachomatis, (vii) swine strains, and (viii) rodent strains of C. trachomatis.

A phylogenetic tree was constructed from the genetic distances determined by the neighbor-joining method (Fig. 2). This tree contained two major clusters of chlamydial strains. The first cluster was composed of five subclusters (avian and ovine bovine abortion strains of $C$. psittaci, guinea pig strains of $C$. psittaci, feline strains of $C$. psittaci, C. pecorum, and $C$. pneumoniae). The second cluster was composed of three subclusters (human strains, swine strains, and rodent strains of $C$. trachomatis). These subclusters corresponded to the genetic 


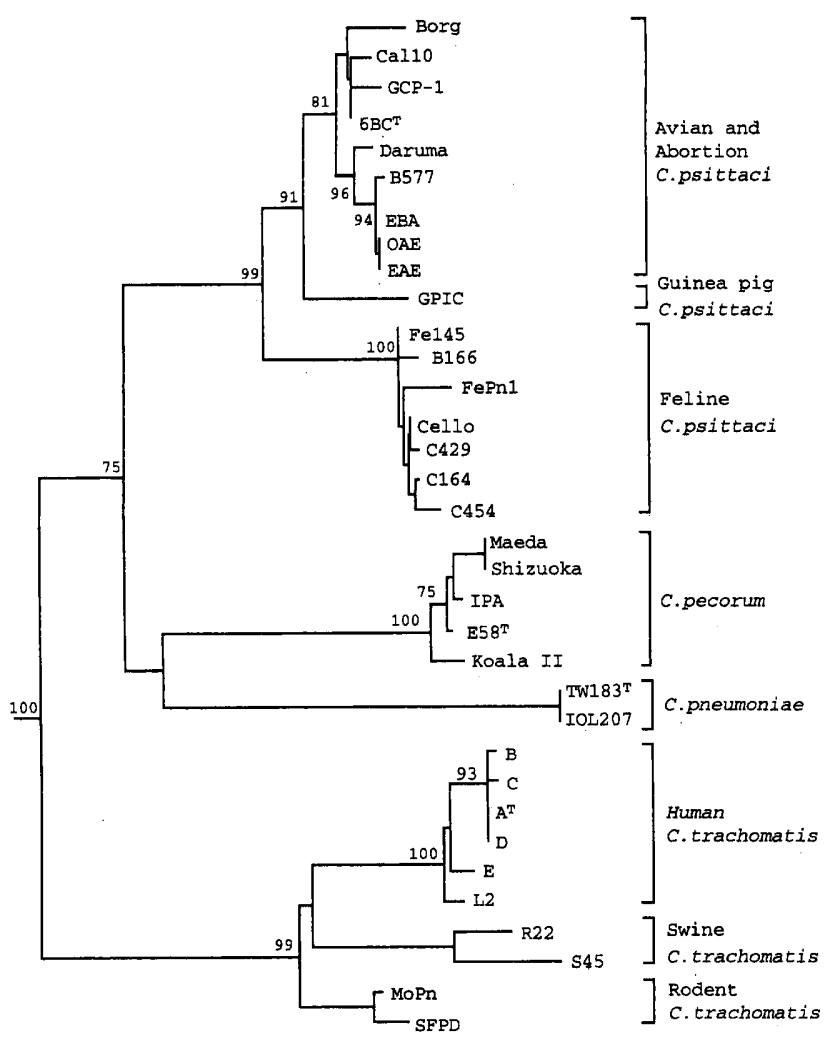

FIG. 2. Phylogenetic tree for Chlamydia spp. based on a sequence analysis of the $16 \mathrm{~S}$ rRNA gene. The tree was constructed by the neighbor-joining method from phylogenetic distances calculated by the Jukes-Cantor method (Table 3 ) The tree root is at the midpoint. The numbers at the branch points are bootstrap values based on 1,000 replicates. Horizontal distances correspond to genetic distances; vertical distances are arbitrary.

groups described above. A bootstrap evaluation was performed to determine the confidence level for the phylogenetic tree.

The sequence of the chlamydia-like organism Simkania sp. strain " $Z$ " was also compared with the sequences of chlamydiae. This sequence exhibited 15.40 to $17.0 \%$ dissimilarity and genetic distances of 0.1685 to 0.1829 compared with the chlamydiae.

\section{DISCUSSION}

A phylogenetic analysis of $16 \mathrm{~S}$ rRNA gene sequences revealed eight groups that formed two clusters within the Chlamydia spp. Each cluster corresponded to a phenotype defined by inclusion morphology, glycogen content of inclusions, sulfadiazine sensitivity, and number of rRNA gene loci. One of the clusters corresponded to $C$. trachomatis, which forms vacuolar inclusions, contains glycogen in its inclusions, is sufladiazine sensitive, and has two rRNA operon loci. The other cluster included $C$. psittaci, $C$. pecorum, and $C$. pneumoniae, which form compact inclusions, contain no glycogen, are sulfadiazine resistant, and have only one rRNA operon locus. The 16S rRNA gene sequences of two strains of $C$. pneumoniae were identical, and the 16S rRNA gene sequences of $C$. pecorum strains were similar. Therefore, the strains of $C$. pneumoniae formed one genetic group, as did the strains of $C$. pecorum. However, strains of both C. psittaci and C. trachomatis fell into three genetic groups.

Our phylogenetic analysis provided a basis for further division of $C$. psittaci into several species. The members of each genetic group of $C$. psittaci had similar biological properties, such as host range, pathogenesis, and biotypes as proposed by Spears and Storz (32). Furthermore, the genetic distances among the $C$. psittaci strains within each genetic group were almost identical to the genetic distances among $C$. trachomatis strains within biovars and the genetic distances among $C$. pecorum strains. These results were also consistent with the previous classification based on biotyping and $o m p A$ allelic analysis data $(23,32)$. Therefore, the genetic groups identified in the present study may be considered species.

It is interesting that the abortion strains were more closely related to avian strains than to feline or guinea pig strains. The close relationship between abortion and avian strains was also revealed by a genomic RFLP analysis (5) and an $o m p A$ allelic analysis (23). This relationship suggests that the abortion strains might have been transmitted from birds to ruminants and adapted to the new hosts. This question may be answered by retrospective epidemiological studies.

Despite the heterogeneity in host origin and in the omp $A$ gene (23), C. pecorum strains seemed to have homogeneous rRNA sequences. When a new species was proposed for $C$. pecorum, the strains came from ruminants, mainly cattle and sheep. At present, pigs and koala strains have been classified as C. pecorum strains. However, no species-specific phenotypic characteristics other than immunological specificity in immunoblotting have been identified. Therefore, the 16S rRNA gene sequence should be a useful marker for identifying $C$. pecorum.

The koala strain tested in the present study was found to belong to $C$. peconum. Two types of koala strains have been recognized $(12,15)$. Type I strains are isolated from conjunctivae, and type II strains are isolated from uteri, vaginas, and recta (12). The two types have been differentiated by restriction enzyme digestion, gene probe analysis, and the presence or absence of a $7.4-\mathrm{kb}$ plasmid $(11,19)$. Our results showed that our koala type II strain was a $C$. pecorum strain. This grouping is consistent with the results of biotyping by the method of Spears and Storz, which showed that a koala type II strain is a biotype 2 strain (15), as are the polyarthritis and sporadic bovine encephalomyelitis isolates of $C$. pecorum. Nucleotide sequence data for the 16S rRNA gene of type I koala strains are not available yet. However, the sequence of the ompA gene of the type I koala strain was similar to the $C$. pneumoniae sequence (23). Further analysis of the 16S rRNA gene sequences of koala strains may clarify the phylogenetic status of these organisms.

The two biovars of human $C$. trachomatis strains formed a single genetic group. The $16 \mathrm{~S}$ rRNA gene sequence of $C$. trachomatis biovar trachoma was similar to that of $C$. trachomatis biovar lymphogranuloma. The results indicated that there is a close relationship between $C$. trachomatis biovar trachoma and $C$. trachomatis biovar lymphogranuloma. The genetic groups of $C$. trachomatis did not seem to correspond to biovar designations. The human $C$. trachomatis strains have two well-defined serological complexes, the $\mathrm{B}$ and $\mathrm{C}$ complexes (33). The sequence analysis did not differentiate between the serological complexes. On the other hand, the sequences of mouse biovar strains and swine biovar strains were different from the sequences of the human biovar strains. The mouse biovar strains, as well as the swine biovar strains, formed a single genetic group.

Many chlamydia-like organisms have been obtained from various vertebrates and invertebrates, including African clawed frogs and turtles $(18,35)$. The PCR amplification and direct sequencing method described in this study may be used to study the molecular relatedness of these chlamydia-like organ- 
isms to chlamydial species. By using these methods the chlamydia-like organism Simkania sp. strain "Z" (22) was shown to be distantly related to the genus Chlamydia.

The variability within $C$. psittaci and $C$. trachomatis was great. However, the variability within genetic groups of the two species was as low as the variability in $C$. pecorum and $C$. pneumoniae. This suggests that the genetic groups within $C$. psittaci and $C$. trachomatis defined in this study may be considered individual species.

In conclusion, genetic grouping should be useful for future classification but should not be the only criterion. Additional studies are needed to consider whether the genetic groups are new species and to construction a classification scheme for the chlamydiae.

\section{ACKNOWLEDGMENTS}

We thank C.-C. Kuo, L. A. Campbell, and A. F. Swanson for their helpful comments and suggestions concerning the manuscript.

This work was supported by grants-in-aid for scientific research 05660342 and 07556120 from the Ministry of Education, Science, Sports and Culture of Japan.

\section{REFERENCES}

1. Devereux, J., P. Haeberli, and O. Smithies. 1984. A comprehensive set of sequence analysis programs for the VAX. Nucleic Acids Res. 12:387-395.

2. Felsenstein, J. 1989. PHYLIP-phylogenetic inference package (version 3.2). Cladistics 5:164-166.

3. Frederick, M. A., R. Brent, R. E. Kingston, D. D. Moore, J. G. Seidman, J. A Smith, and K. Struhl. 1989. Current protocols in molecular biology. Greene Publishing Associates and Wiley-Interscience, New York, N.Y.

4. Fukushi, H., and K. Hirai. 1988. Immunochemical diversity of the major outer membrane protein of avian and mammalian Chlamydia psittaci. J. Clin. Microbiol. 26:675-680.

5. Fukushi, H., and K. Hirai. 1989. Genetic diversity of avian and mammalian Chlamydia psittaci strains and relation to host origin. J. Bacteriol. 171:28502855

6. Fukushi, H., and K. Hirai. 1992. Proposal of Chlamydia peconum sp. nov. for Chlamydia strains derived from ruminants. Int. J. Syst. Bacteriol. 42:306-308

7. Fukushi, H., and K. Hirai. 1993. Chlamydia pecorum - the fourth species of genus Chlamydia. Microbiol. Immunol. 37:515-522.

8. Fukushi, H., and K. Hirai. 1993. Restriction fragment length polymorphisms of rRNA as genetic markers to differentiate Chlamydia spp. Int. J. Syst. Bacteriol. 43:613-617.

9. Gaydos, C. A., T. C. Quinn, and J. J. Eiden. 1992. Identification of Chlamydia pneumoniae by DNA amplification of the $16 \mathrm{~S}$ rRNA gene. J. Clin. Microbiol 30: $796-800$.

10. Gaydos, C. A., L. Palmer, T. C. Quinn, S. Falkow, and J. J. Eiden. 1993. Phylogenetic relationship of Chlamydia pneumoniae to Chlamydia psittaci and Chlamydia trachomatis as determined by analysis of $16 \mathrm{~S}$ ribosomal DNA sequences. Int. J. Syst. Bacteriol. 43:610-612.

11. Girjes, A. A., A. F. Hugall, P. Timms, and M. F. Lavin. 1988. Two distinct forms of Chlamydia psittaci associated with disease and infertility in Phascolarctos cinereus (koala). Infect. Immun. 56:1897-1900.

12. Girjes, A. A., A. Hugall, D. M. Graham, T. F. McCaul, and M. F. Lavin. 1993. Comparison of type I and type II Chlamydia psittaci strains infecting koalas (Phascolarctos cinereus). Vet. Microbiol. 37:65-83.

13. Gordon, F. B., and A. L. Quan. 1965 . Occurrence of glycogen in inclusions of the psittacosis-lymphogranuloma venerum-trachoma agents. J. Infect. Dis. 115:186-196

14. Grayston, J. T., C.-C. Kuo, L. A. Campbell, and S. Wang. 1989. Chlamydia pneumoniae sp. nov. for Chlamydia sp. strain TWAR. Int. J. Syst. Bacteriol. 39:88-90.

15. Grice, R. G., and A. S. Brown. 1984. A tissue culture procedure for the isolation of Chlamydia psittaci from koalas (Phascolarctos cinereus). Aust. J. Exp. Biol. Med. Sci. 63:283-286.

16. Gutell, R. R. 1994. Collection of small subunit (16S- and 16S-like) ribosomal RNA structures: 1994. Nucleic Acids Res. 22:3502-3507.

17. Higgins, D. G., and P. M. Sharp. 1990. Fast and sensitive multiple sequence alignments on a microcomputer. Comput. Applic. Biosci. 5:151-153.

18. Homer, B. L., E. R. Jacobson, J. Schumacher, and G. Scherba. 1994 Chlamydiosis in mariculture-reared green sea turtles (Chelonia mydas). Vet. Pathol. 31:1-7.

19. Hugall, A., P. Timms, A. A. Girjes, and M. F. Lavin. 1989. Conserved DNA sequences in chlamydial plasmid. Plasmid 22:91-98.

20. Ina, Y. 1994. ODEN: a program package for molecular evolutionary analysis and database search of DNA and amino acid sequences. Comput. Applic. Biosci. 10:11-12.

21. Jukes, T. H., and C. R. Cantor. 1969. Evolution of protein molecules, p. 21-132. In H. N. Munro (ed.), Mammalian protein metabolism. Academic Press, Inc., New York, N.Y.

22. Kahane, S., E. Metzer, and M. G. Friedman. 1995. Evidence that the novel microorganism ' $Z$ ' may belong to a new genus in the family Chlamydiaceae. FEMS Microbiol. Lett. 126:203-208.

23. Kaltenboeck, B., K. G. Kousoulas, and J. Storz. 1993. Structures of allelic diversity and relationships among the major outer membrane protein (ompA) genes of the four chlamydial species. J. Bacteriol. 175:487-502.

24. Krieg, N. R., and J. G. Holt (ed.). 1984. Bergey's manual of systematic bacteriology, vol. 1. The Williams \& Wilkins Co., Baltimore, Md

25. Moulder, J. W., T. P. Hatch, C.-C. Kuo, J. Schachter, and J. Storz. 1984 Genus I. Chlamydia Jones, Rake and Stearns 1945 , 55 $5^{\mathrm{AL}}$, p. $729-739$. In N. R. Krieg and J. G. Holt (ed.), Bergey's manual of systematic bacteriology, vol. 1. The Williams \& Wilkins Co., Baltimore, Md.

26. Page, L. A. 1968. Proposal of the recognition of two species in the genus Chlamydia Jones, Rake, and Stearns, 1945. Int. J. Syst. Bacteriol. 18:51-66.

27. Saitou, N., and M. Nei. 1987. The neighbor-joining method: a new method for reconstructing phylogenetic trees. Mol. Biol. Evol. 4:406-425.

28. Sanger, F., S. Nicklen, and A. R. Coulson. 1977. DNA sequencing with chain-terminating inhibitors. Proc. Natl. Acad. Sci. USA 74:5463-5467.

29. Selander, R. K., D. A. Caugant, and H. Ochman. 1986. Methods for multilocus enzyme electrophoresis for bacterial population genetics and systematics. Appl. Environ. Microbiol. 51:873-884.

30. Show, N. 1974. Lipid composition as guide to the classification of bacteria, $p$ 63-108. In D. Perlman (ed.), Advances in applied microbiology. Academic Press, London, United Kingdom.

31. Sneath, P. H. A., and R. R. Sokal. 1973. Numerical taxonomy: the principle and practice of numerical classification. W. H. Freeman, San Francisco Calif.

32. Spears, P., and J. Storz. 1979. Biotyping of Chlamydia psittaci based on inclusion morphology and response to diethylaminoethyl-dextran and cycloheximide. Infect. Immun. 24:224-232.

33. Wang, S. P., and J. P. Grayston. 1982. Microimmunofluorescence antibody responses in Chlamydia trachomatis infection, a review, p. 301-315. In The 5th International Symposium on Human Chlamydial Infections, vol. 2 Elsevier Biomedical Press, Amsterdam, The Netherlands.

34. Weisburg, W. G., T. P. Hatch, and C. R. Woese. 1986. Eubacterial origin of chlamydiae. J. Bacteriol. 167:570-574.

35. Wicke, B. M., C. E. Newcomer, M. R. Anver, and G. W. Nace. 1983, Isolation of Chlamydia psittaci from naturally infected African clawed frogs (Xenopus laevis). Infect. Immun. 41:789-794.

36. Woese, C. R. 1987. Bacterial evolution. Microbiol. Rev. 51:221-271. 